\title{
Threat Related Attention and Coping with Lower Limb Amputation
}

\author{
A. Gilad MD", E Keltz MD $^{2}$, D Shamai-Leshem MA $^{3}$, Y Bar-Haim PhD ${ }^{3}$, Y Keren ${ }^{2 *}$ MD and E \\ Fruchter $\mathrm{MD}^{4,5 * *}$ \\ ${ }^{1}$ Orthopedic Division, Shamir Health care campus, Be'er Ya'akov, Israel. And Sackler Medical school, Tel-Aviv University, Israel \\ ${ }^{2}$ Orthopedic Division, Rambam Health care campus, Haifa, Israel \\ ${ }^{3}$ School of Psychological Sciences and Sagol School of Neuroscience, Tel Aviv University, Tel Aviv, Israel \\ ${ }^{4}$ Psychiatric Division, Rambam Health care campus, Haifa, Israel \\ ${ }^{5}$ Ruth and Brus Rapaport faculty of medicine, Technion, Haifa, Israel \\ *Reflecting equal contribution of the two last authors \\ **Corresponding author: Eyal Fruchter, Psychiatry \& Mental Health Division, Rambam Health Care Campus, Haifa, Israel. And Ruth \\ and Brus Rapaport faculty of medicine, Technion, Haifa, Israel, B.0. 9602, Haifa, Israel 3109601, +972529205905, +97247773050, \\ e_fruchter@rmc.gov.il.
}

To Cite This Article: E Fruchter, Threat Related Attention and Coping with Lower Limb Amputation. 2020 - 8(2). AJBSR.MS.ID.001241.

DOI: 10.34297/AJBSR.2020.08.001241.

Received: 泚 March 09, 2020; Published: 眥 March 12, 2020

\section{Abstarct}

Background: It is fairly common practice for adult patients to undergo amputation of a lower limb as a complication of diabetes mellitus neuropathy. Coping with such a dramatic and potentially lethal event is crucial for future physical and mental health. There is no reliable method for evaluating coping differences amongst patients. Our aim was to examine a potential tailor-made evaluation tool based on attention differences.

Methods: A Cognitive test of Attention Bias was transferred using the Double Dot Probe Task (DDPT) amongst diabetic patients in need for amputation. The test is differentiating between "avoidant" and "anxious" coping styles. A prospective follow-up was conducted using The Katz Questionnaire, healing of the surgical stump, Hgb-A1C values and whether prosthesis had been adapted. The relationship between the attention bias tests and the indices examined was tested to assess the ability to cope with the new condition.

Results: 16 pre-amputation diabetic patients were evaluated. Those found to cope "avoidantly" were older than those coping "anxiously". The average hospitalization duration was shorter amongst those with an avoidant coping style. Deteriorated daily functioning was recorded in the anxious group. From an aspect of the post-operative Hgb-A1C values, the results were not consistent.

Conclusions: there is an important difference in coping and outcome, between the two groups. In order to determine whether DDPT software enables us to evaluate patient's ability to cope with the amputation, we need to expand the patient groups and adapt the software to the patients' ability.

Keywords: DDPT; Katz; ADL; Amputation

Abbreviations: DDPT: Double Dot probe task; ADL: Activity of Daily Living; Hgb A1C: Hemoglobin A1C; PTSD: Post Traumatic Stress Disorder; IDF: Israeli Defense Force; AK: Above Knee; BK: Bellow Knee

\section{Introduction}

Patients with infected diabetic ulcers or peripheral ischemia with spreading gangrene often require limb amputation. Limb amputation, although medically crucial at times, is associated with sort and long terms high mortality rates. Specifically, 30 days' mortality of the patients following a lower limb amputated is approximately $25 \%$. Median survival is estimated at around 20 months, with 1 -year and 5 -years survival rates of $56 \%$ and $23 \%$, respectively [1]. There is a well-established link between the psychological reaction of patients to the tidings of the need for amputation and the success of recovery from the surgical procedure 
and coping with its results. Parkes et al. [2] projected Kobler Ros' [3] five stages of grief onto dealing with amputation: Denial, anger, bargaining, depression, and acceptance. Holmes and Rahe [4] described the grief process suggest that increased and exceptional emotional response during grief may prevent the patient from recovering due to profound physical and emotional morbidity.

Emotional response to threat in the forms of anxiety, depression, posttraumatic stress symptoms, and pain has been associated with biased attentional processing of threat-related information [5-7] The dot probe task [8] is a widely used to measure of threat-related attention biases [9-11]. In this task, two stimuli, one threat-related and one neutral, are simultaneously shown on each trial, and their offset is followed by a small probe appearing at the location just occupied by one of the stimuli. Participants are asked to indicate probe position as fast as possible without compromising accuracy. Response latencies on the dot-probe task are held to provide a "snapshot" of the distribution of participants' attention, with faster responses to probes presented in the attended relative to the unattended location. Attentional bias toward threat is inferred when participants are faster to respond to probes that replace threat rather than neutral stimuli whereas the reverse pattern is thought to reflect threat avoidance [12].

Attentional threat avoidance measured by the dot-probe task emerged as predicative of susceptibility to PTSD following combat exposure in deploying Israel Defense Force soldiers [13] and US Army soldiers [14] Further research suggests that attentional training designed to reduce attentional threat avoidance can reducing risk for PTSD symptoms following deployment $[15,16]$.

In the current pilot study, we preliminarily characterize threatrelated attention patterns of orthopedic patients planned for lower limb amputation and evaluate how these attention patterns relate to patients' ability to adjust to life post the surgical procedure.

\section{Research Assumptions}

Patients with baseline attentional threat avoidance would:

1. Display greater trouble accepting the need for limb amputation.

2. Struggle to adapt to the new situation following the surgical procedure and their rehabilitation will be slower, as reflected in the KATZ questionnaire scores (Appendix 1).

3. Struggle to remain healthy, balance diabetes, and would have higher values of $\mathrm{Hgb}-\mathrm{A} 1 \mathrm{C}$ and more stump complications.

\section{Materials and Methods}

Our research is a prospective, analytic, and observational.

\section{Participants}

16 adult patients hospitalized in the Orthopedic Department at the Rambam Hospital in Haifa, Israel, for the purpose of a lower limb amputation. Inclusion criteria: a) Need for one lower limb amputation surgery as a complication of diabetes mellitus alone or in combination with a vascular disease; b) a cooperative patient, time and place oriented; c) 18 years of age or older. Exclusion criteria: a) need for amputation based on a vascular disease alone b) lack of diabetes mellitus; c) a former limb amputation; d) a psychiatric background; e) chronic use of drugs or alcohol; a traumatic cause for amputation; f) Buerger disease as a cause for amputation; e) a need for two limb amputation.

All the patients met the research inclusion criteria and provided a signed informed consent. The research was approved by the Rambam Hospital Ethics Comity.

\section{Methods and Measures}

Attention to threat was assessed using 120 trials of the angryneutral faces dot probe task (TAU-NIMH ABMT initiative; http://tau. ac.il/ yair1/ABMT.html). Sequence of events on a dot-probe trial is depicted in Appendix 2. In each trial, a fixation cross appeared first $(500 \mathrm{~ms})$, followed by two faces of the same actor $(500 \mathrm{~ms})$, one above the other. One face depicted an angry expression and the other depicted a neutral expression. Then a probe (: or.) appeared in the location of one of the faces. Participants indicated the type of probe by pressing either the left or the right mouse button. The probe remained on the screen until response. After responding, the next trial began. Consistent with past studies, attention to threat was calculated as the difference in response times to probes appearing in the location of the angry face relative to the neutral face. Positive scores indicate attention toward threat (vigilance) whereas negative scores reflect attentional avoidance. The task ran E-Prime software (E-Prime, Psychology software tools, PA, USA).

Participants completed the dot-probe task at baseline, shortly before the time of limb amputation. During the patient's hospitalization period, their ability to perform daily living activity was assessed using the KATZ questionnaire (Appendix 1). Based on this questionnaire a score of $6=$ full independence, $4=$ medium decline in functioning; and 2 or less = severe decline in the functioning in daily routine activity.

The following data was collected regarding each patient: sex, age, marital status, date of arrival at the department, date of surgery, affected limb side, height of amputation - AK (above knee) or BK (below knee), the culprit disease, Hgb-A1C prior to surgery, ASA score- American Society of Anesthesiologists' score a presurgery physical status classification system, date of release from the department. Three months after surgery all patients visited the clinic and completed the KATZ questionnaire again. Their healing of the surgical stump was inspected and updated Hgb-A1C values were taken. Finally, whether a prosthesis had been adapted was noted. When a patient died during the surveillance period, his data were included until the point of his death, if we had the base line data. 


\section{Statistical Analysis}

Descriptive statistics were extracted to describe the distribution of demographic variables and morbidity. Continuous variables such as length of hospitalization, $\mathrm{Hgb}-\mathrm{A} 1 \mathrm{C}$, age, and ordinal variables such as the KATZ questionnaire score were described using average, range, median, and standard deviation. A comparison of age, length of hospitalization, KATZ score, and Hgb-A1C was made among the patients, who, during the 3 months' post-surgery, had no medical complications compared with those who had some medical complication, including death. A comparison was made using a Mann-Whitney Non-Parametric Test.

Evaluating patients' attention bias parameters and other variables such as the cause of surgery, ASA score, pre-surgery KATZ score, pre-surgery Hgb-A1C, and whether or not a medical complication was encountered during the 3 months follow-up, was computed using double-variable logistic regression - odd ratio and $\mathrm{p}$ value are reported. Data analysis was conducted using SPSS for Windows version 21 with the assistance of a statistical analyst of the Rambam Hospital in Haifa.

\section{Results}

Of the 16 patients enrolled in the study 14 provided data at the 3-months follow-up. In addition, attention bias scores of participants were not available due to technical failure. Thus, all reported analyses were conducted on the 13 patients who provided complete data sets.

\section{Patients' Demographic Data}

Patients' demographic and clinical data are described in Table

\begin{tabular}{|c|c|c|c|c|c|c|c|c|c|c|c|}
\hline & & Pts. & w/o Complication & & & & $95 \% \mathrm{CI}$ & & & Died & \\
\hline & & No. & $\%$ from total & No. & $\%$ & $P$ value & OR & Lower & Upper & No. & $\%$ \\
\hline & Total & 13 & $10 \mathrm{a} 0 \%$ & 6 & 46.2 & . & . & . & . & 3 & 23.1 \\
\hline \multirow[t]{2}{*}{ Gender } & $\mathrm{F}$ & 1 & $8 \%$ & 1 & 100 & . & 1 & . & . & 0 & 0 \\
\hline & M & 12 & $92 \%$ & 5 & 41.7 & 1 & 0 & 0 & 0 & 3 & 25 \\
\hline \multirow[t]{3}{*}{ Age } & $<60$ & 4 & $31 \%$ & 2 & 50 & 0.587 & 1 & 0 & 0 & 1 & 25 \\
\hline & $60-69$ & 4 & $31 \%$ & 1 & 25 & 0.472 & 0.33 & 0.02 & 6.65 & 1 & 25 \\
\hline & $70+$ & 5 & $38 \%$ & 3 & 60 & 0.765 & 1.5 & 0.11 & 21.31 & 1 & 20 \\
\hline \multirow[t]{2}{*}{ Surgery Type } & AKA & 1 & $8 \%$ & 0 & 0 & . & 1 & . & . & 1 & 100 \\
\hline & BKA & 12 & $92 \%$ & 6 & 50 & 1 & 16154750 & 53.72 & 0 & 2 & 16.7 \\
\hline \multirow[t]{2}{*}{ Surgery Side } & Rt. & 3 & $23 \%$ & 1 & 33.3 & . & 1 & . & . & 1 & 33.3 \\
\hline & Lt. & 10 & $77 \%$ & 5 & 50 & 0.615 & 2 & 0.13 & 29.81 & 2 & 20 \\
\hline \multirow[t]{2}{*}{ Cause } & Diabetes & 6 & $46 \%$ & 3 & 50 & . & 1 & . & . & 2 & 33.3 \\
\hline & Combined & 7 & $54 \%$ & 3 & 42.9 & 0.797 & 0.75 & 0.08 & 6.71 & 1 & 14.3 \\
\hline \multirow[t]{3}{*}{ ASA Score } & 2 & 2 & $15 \%$ & 1 & 50 & 0.99 & 1 & 0 & 0 & 1 & 50 \\
\hline & 3 & 9 & $69 \%$ & 5 & 55.6 & 0.887 & 1.25 & 0.06 & 26.87 & 2 & 22.2 \\
\hline & 4 & 2 & $15 \%$ & 0 & 0 & 0.999 & 0 & 0 & 0 & 0 & 0 \\
\hline$\Delta$ Katz & $0-2$ & 3 & $23 \%$ & 0 & 0 & & & & & 0 & 0 \\
\hline \multicolumn{12}{|l|}{ Pre-surgery } \\
\hline & 4-Mar & 4 & $31 \%$ & 3 & 75 & & & & & 1 & 25 \\
\hline & 6-May & 6 & $46 \%$ & 3 & 50 & & & & & 2 & 33.3 \\
\hline Hgb-A1C & $\leq 6.5$ & 4 & $31 \%$ & 1 & 25 & . & 1 & . & . & 1 & 25 \\
\hline \multicolumn{12}{|l|}{ Pre-surgery } \\
\hline . & $>6.5$ & 9 & $69 \%$ & 5 & 55.6 & 0.322 & 3.75 & 0.27 & 51.37 & 2 & 22.2 \\
\hline Katz & $0-2$ & 8 & $62 \%$ & 5 & 62.5 & 1 & 1 & 0 & 0 & 0 & 0 \\
\hline \multicolumn{12}{|l|}{ Post-surgery } \\
\hline & 4-Mar & 1 & $8 \%$ & 1 & 100 & 1 & 96928490 & 5.71 & 0 & 0 & 0 \\
\hline & 6-May & 2 & $15 \%$ & 0 & 0 & 0.999 & 0 & 0 & 0 & 1 & 50 \\
\hline & Missing & 2 & $15 \%$ & 0 & 0 & . & . & . & . & 2 & 100 \\
\hline
\end{tabular}




\begin{tabular}{|c|c|c|c|c|c|c|c|c|c|c|c|}
\hline $\begin{array}{c}\text { HGb-A1C } \\
\text { Post-surgery }\end{array}$ & $\leq 6.5$ & 3 & $23 \%$ & 2 & 66.7 & . & 1 & . & . & 0 & 0 \\
\hline & $>6.5$ & 5 & $38 \%$ & 2 & 40 & 0.472 & 0.33 & 0.02 & 6.65 & 1 & 20 \\
\hline & Missing & 5 & $38 \%$ & 2 & 40 & . & . & . & . & 2 & 40 \\
\hline \multirow[t]{4}{*}{$\Delta$ Katz } & - 2 cat. & 2 & $15 \%$ & 2 & 100 & . & . & . & . & 0 & 0 \\
\hline & - 1 cat. & 4 & $31 \%$ & 4 & 100 & . & . & . & . & 0 & 0 \\
\hline & w/o change & 5 & $38 \%$ & 0 & 0 & . & . & . & . & 1 & 20 \\
\hline & Missing & 2 & $15 \%$ & 0 & 0 & . & . & . & . & 2 & 100 \\
\hline \multirow[t]{3}{*}{ Outcome } & $\begin{array}{l}\text { w/o compli- } \\
\text { cation }\end{array}$ & 6 & $46 \%$ & 6 & 100 & . & . & . & . & 0 & 0 \\
\hline & Complication & 4 & $31 \%$ & 0 & 0 & . & . & . & . & 0 & 0 \\
\hline & Died & 3 & $23 \%$ & 0 & 0 & . & . & . & . & 3 & 100 \\
\hline
\end{tabular}

For 12 patients it was decided by the orthopedic team that the appropriate surgery would be BK amputation, while 1 needed an AK amputation. The cause for amputation of 6 patients was diabetes mellitus alone, while 7 patients had a combination of diabetes mellitus and vascular disease at the time of surgery. At the 3-months follow-up, it was determined that the stump wound of 5 patients had not healed adequately, while for 5 patients in was completely healed. 1 patient refused to come for a follow up, 2 died prior to the visit. The patients' ASA score was set by anesthesiologists prior to surgery. Two patients had an ASA score of 2, nine had an ASA score of 3, and two had an ASA score of 4. An assessment of the ADL was made using the KATZ questionnaire, before and after the surgery.

Two of the patients had a 2-point decline on their KATZ score; four had a 1- point decline, while, for five patients, the KATZ score did not change as result of the surgery. Out of the participating 13 patients, 3 died during the 3 months' follow-up of the research (one died 2 weeks following his discharge from the hospital, the second died after 3 months and the third 4 months following his discharge, while during those months he had a revision surgery and had to change his BK amputation to an AK amputation). 4 patients had complications regarding the surgery and the amputated limb, but they managed to survive those complications. 5 patients had to have additional surgery (revision). Out of those 5, one died during the follow-up period, 2 patients had an $\mathrm{AK}$ amputation during follow-up, on the same side of the BK amputation (one died), and two more patients had an amputation of the other limb. In four cases, no complications were witnessed during follow-up, while two patients were lost to follow-up.

Three patients had a prosthesis adapted to the amputated limb, two other patients were noted to have a stump in a good condition, allowing a prosthesis to be adjusted. Results of the analysis were statistically processed using the Mann -Whitney U test, in order to adjust age, time of hospital stay, KATZ questionnaire and Hgb-A1C pre- and post-surgery (Table 2).

Table 2: Reaction time between 200 and 3500 milliseconds. RT-Reaction time, SD-standard Deviation, LOS-Length of Stay, Hgb-A1C- Hemoglobin A1C.

\begin{tabular}{|c|c|c|c|c|c|c|c|c|}
\hline & Attention Pattern & No. & Mean & SD & Median & Min. & Max. & $P$ value \\
\hline \multirow[t]{2}{*}{ Age } & Avoidant & 7 & 70.6 & 6.9 & 68 & 61 & 81 & 0.252 \\
\hline & Vigilant & 6 & 65 & 14.9 & 58.5 & 50 & 88 & . \\
\hline \multirow[t]{2}{*}{ LOS } & Avoidant & 7 & 23.4 & 6.9 & 21 & 18 & 38 & 0.252 \\
\hline & Vigilant & 6 & 32 & 37.7 & 15 & 9 & 107 & . \\
\hline Katz & Avoidant & 7 & 4 & 1.9 & 3 & 2 & 6 & 0.495 \\
\hline \multicolumn{9}{|l|}{ Pre-surgery } \\
\hline & Vigilant & 6 & 4.7 & 1.6 & 5 & 2 & 6 & . \\
\hline Katz & Avoidant & 6 & 3 & 2.4 & 2 & 1 & 6 & 0.449 \\
\hline \multicolumn{9}{|l|}{ Post-surgery } \\
\hline & Vigilant & 5 & 1.6 & 1.1 & 2 & 0 & 3 & . \\
\hline \multirow[t]{2}{*}{$\Delta$ Katz } & Avoidant & 6 & -1.2 & 1.5 & -1 & -4 & 0 & 0.072 \\
\hline & Vigilant & 5 & -2.8 & 1.3 & -3 & -4 & -1 & . \\
\hline HGb-A1C & Avoidant & 7 & 7.2 & 1.6 & 6.5 & 5.7 & 9.3 & 0.431 \\
\hline \multicolumn{9}{|l|}{ Pre-surgery } \\
\hline & Vigilant & 6 & 7.6 & 0.6 & 7.6 & 6.8 & 8.6 & . \\
\hline
\end{tabular}




\begin{tabular}{|c|c|c|c|c|c|c|c|c|}
\hline HGb-A1C & Avoidant & 5 & 6.6 & 1.5 & 5.9 & 5.2 & 8.5 & 0.18 \\
\hline Post-surgery & & & & & & & & \\
\hline & Vigilant & 3 & 8.2 & 1.2 & 8.3 & 7 & 9.3 &. \\
\hline AHGb-A1C & Avoidant & 5 & -1.2 & 1.7 & -1.3 & -3.8 & 0.8 & 0.072 \\
\hline & Vigilant & 3 & 0.8 & 0.8 & 0.8 & 0 & 1.5 &. \\
\hline
\end{tabular}

Seven patients had shown threat-related avoidance, whereas 6 patients had shown threat vigilance. None of the between-groups comparisons were statistically significant. However, it may be worth noting two non-significant trend level results. First, relative to the attentionally vigilant patients, avoidant patients seem to have had a smaller decline in functioning from pre-surgery to followup (Katz scores). Second, it seems that the attentionally avoidant patients were better able to regulate their sugar levels $(\Delta \mathrm{Hgb}-\mathrm{A} 1 \mathrm{C}$ values) relative to the vigilant patients.

\section{Discussion}

Lower limb amputation due to diabetes and vascular disease complications is a common procedure. A post- surgery follow-up of these patients reveals that a significant percentage requires additional interventions, and many are unable to deal with the new situation. To the best of our knowledge, factors related to patients' psychological character and cognitive processing style are not taken into consideration by healthcare providers. Here we tested whether threat-related attention biases recorded at pre-amputation relate to patient's recovery from a limb amputation. The main limitation of the study stems from its small sample size, therefore, all the findings should be taken with a limited confidence.

Our main finding showed that avoidant attentional pattern seems more adaptive for the patients after amputation. This finding is supported by the work of Sheppes et al. [17] showing that under extreme threat people usually select to regulate their emotion using avoidance strategies rather than re- appraisal ones. Having to go through amputation is an extreme medical and functional threat to all people and is harder for older people suffering from severe diabetes. It makes sense that a vigilant strategy would cause less control of the Insulin and steroids, all stress hormones, and cause a deteriorated diabetic control as well as lower pain and sensation threshold thus making the amputation wound healing and using artificial limb less possible.

After analyzing the results at various reaction time (200$3000 \mathrm{~ms}, 200-3500 \mathrm{~ms}, 200 \mathrm{~ms}$ to an unlimited time, not detailed in the text), we have found that the most suitable reaction time for this purpose is between 200 and $3500 \mathrm{~ms}$. The reason is that in this range, the two groups are quantitatively equal, and a more significant different can be observed regarding the decline in the ADL index (calculated using the KATZ questionnaire) and the change in Hgb-A1C. The small number of patients precipitating in the research caused the results to be insignificant statistically.
Nevertheless, the consistent differences are in favor of the more avoidant group, leading to an assumption that a larger study would produce significant results.

We recommend that in an extended research, an ASA score of 4 or more should be an exclusion criterion, since the only 2 patients with this score, have had post-surgery complications. Despite the small number of patients, we think that a high ASA score puts the patient at risk of complications regardless of the software results.

The potential use of cognitive processing of threat indices in the context of limb amputation could be an important step, on the root for their post-operative rehabilitation and survival. It may be found, as suggested in this small sample research, that training people to avoid threat in this stage could prove beneficial to their recovery. As mentioned earlier, the research has several limitations is small sample size, the difficulty to operate the dot- probe task by some of the patients, reliance on self- report questionnaire rather than an objective functioning test etc. However, despite tie low evidence of effect in the current study it appears that the extant trend level finding does appear to warrant additional research with a larger sample.

\section{Conclusions}

It is crucial to find tools to evaluate the mental and cognitive coping of people undergoing a major medical procedure such as amputation. Due to those findings, along with the long reaction times, we concluded that the DDPT as currently exists, is unsuitable for the population chosen for the research, and needs to be adjusted. We believe that, in order to evaluate and supply these patients with better physical and emotional resources, we would need to find an easier cognitive tool for evaluation. Our preliminary results presented here, may serve as a steppingstone to a larger more controlled and elderly suited study. We estimate that trauma patients arriving at the Orthopedic Department could be a better research group due to their younger age. This group is thought to be more homogenous, due to fewer background diseases, and fewer variables interfering with statistical comparisons. Younger patients are probably more skilled in computer use and would be able to operate the software significantly faster.

\section{Acknowledgement}

This research could not have been completed without the help of an important individual. We would like to thank Ms. Tanya Mashiach, a statistical analyst of the Rambam Hospital in Haifa. 


\section{Conflict of Interest}

The authors declare no conflict of interest regarding the writing of this paper.

\section{References}

1. Fortington LV, Geertzen JHB, van Netten JJ, Postema K, Rommers GM, et al. (2013) Short- and Long-Term Mortality Rates after a Lower Limb Amputation. Eur J Vasc Endovasc Surg 46(1): 124-131.

2. Parkes CM (1975) Psycho-social Transitions: Comparison between Reactions to Loss of a Limb and Loss of a Spouse. Br J Psychiatry 127: 204-210.

3. Kübler Ross E (1969) On death and dying.

4. Holmes TH, Rahe RH (1967) The Social Readjustment Rating Scale. J Psychosom Res 11(2): 213-218.

5. Bar Haim Y, Lamy D, Pergamin L, Bakermans Kranenburg MJ, Van Ijzendoorn MH (2007) Threat-related attentional bias in anxious and nonanxious individuals: A meta-analytic study. Psychol Bull 133(1): $1-24$.

6. Crombez G, Van Ryckeghem DML, Eccleston C, Van Damme S (2013) Attentional bias to pain-related information: A meta-analysis. Pain 154(4): 497-510.

7. Peckham AD, McHugh RK, Otto MW (2010) A meta-analysis of the magnitude of biased attention in depression. Depress Anxiety 27(12): 1135-1142.

8. MacLeod C, Mathews A, Tata P (1986) Attentional bias in emotional disorders. J Abnorm Psychol 95(1): 15-20.

9. Bryant RA, Harvey AG (1997) Attentional bias in posttraumatic stress disorder. J Trauma Stress 10(4): 635-644.
10. Bradley, Mogg K, White J, Groom C, de Bono J (1999) Attentional bias for emotional faces in generalized anxiety disorder. Br J Clin Psychol 38(3): 267-278.

11. Thoern HA, Grueschow M, Ehlert U, Ruff CC, Kleim B (2016) Attentional bias towards positive emotion predicts stress resilience. PLoS One 11(3): 0148368.

12. Iacoviello BM, Wu G, Abend R, Murrough JW, Feder A, et al. (2014) Attention Bias Variability and Symptoms of Posttraumatic Stress Disorder. J Trauma Stress 27(2): 232-239.

13. Wald I, Degnan KA, Gorodetsky E, Charney DS, Fox NA, et al. (2013) Attention to threats and combat-related posttraumatic stress symptoms: prospective associations and moderation by the serotonin transporter gene. JAMA psychiatry 70(4): 401-408.

14. Sipos ML, Bar Haim Y, Abend R, Adler AB, Bliese PD (2014) Postdeployment threat-related attention bias interacts with combat exposure to account for PTSD and anxiety symptoms in soldiers. Depress Anxiety 31(2): 124-129.

15. Wald I, Fruchter E, Ginat K, Stolin E, Dagan D, et al. (2016) Selective prevention of combat-related post-traumatic stress disorder using attention bias modification training: a randomized controlled trial. Psychol Med 46(1): 2627-2636.

16. Wald I, Bitton S, Levi O, Zusmanovich S, Fruchter E, et al. (2017) Acute delivery of attention bias modification training (ABMT) moderates the association between combat exposure and posttraumatic symptoms: A feasibility study. Biol Psychol 122: 93-97.

17. Sheppes G, Suri G, Gross JJ (2015) Emotion Regulation and Psychopathology. Annu Rev Clin Psychol 11: 379-405. 